\title{
Coherence and anticoherence resonance in high-concentration erbium-doped fiber laser
}

\author{
Sergey Sergeyev, ${ }^{1,2, *}$ Kieran O’Mahoney, ${ }^{1}$ Sergei Popov, ${ }^{3}$ and Ari T. Friberg ${ }^{3,4,5}$ \\ ${ }^{1}$ Waterford Institute of Technology, Optics Research Group, Cork Road, Waterford, Ireland \\ ${ }^{2}$ School of Engineering \& Applied Science, Aston University, Birmingham, B4 7ET, UK \\ ${ }^{3}$ Royal Institute of Technology, Department of Microelectronics and Applied Physics, Electrum 229, SE-164 40 Kista, Sweden \\ ${ }^{4}$ Aalto University, Department of Applied Physics, FI-00076 Aalto, Finland \\ ${ }^{5}$ University of Eastern Finland, Department of Physics and Mathematics, FI-80101 Joensuu, Finland \\ *Corresponding author: sergey.sergeyev@gmail.com
}

Received August 2, 2010; revised September 21, 2010; accepted September 26, 2010; posted October 7, 2010 (Doc. ID 132698); published November 3, 2010

\begin{abstract}
We report an experimental study of low-frequency $(\sim 10 \mathrm{kHz})$ self-pulsing of the output intensity in a highconcentration erbium-doped fiber laser. We suggest that the fast intensity fluctuations caused by multimode and polarization instabilities play the role of an external noise source, leading to low-frequency auto-oscillations through a coherence resonance scenario. (c) 2010 Optical Society of America

OCIS codes: $\quad 060.3510,060.2410,140.3500,140.1540$.
\end{abstract}

Output intensity self-pulsing in high-concentration erbium-doped fiber lasers (HC EDFLs) has been extensively studied for its importance in communications, reflectometry, distributed fiber-optic sensing, biomedicine, etc. [1-7]. For a long time, the presence of clustered erbium ions and their role as saturable absorber was regarded as the only possible mechanism responsible for self-pulsing at frequencies of $10-100 \mathrm{kHz}$ [7]. However, detailed microscopic study of erbium-doped glasses by means of x-ray-absorption fine structure spectroscopy (XAFS) has revealed a short-range coordination order of erbium ions, rather than pair clustering []. It was also found that suppression of the short-range order leads to improved characteristics of high-concentration erbiumdoped fiber amplifiers and lasers. The suppression can be realized by increasing the solubility of erbium in the host matrix (codoping by $\mathrm{Al}$ [9]) or by changing the deposition process (direct nanoparticle deposition $[10,11])$. Because self-pulsing was observed in HC EDFLs even with these types of fibers, the pair-clusters approach cannot explain this effect $[4,5]$. On the other hand, experiments and theory indicate that the pump-tosignal intensity noise transfer (PSINT) can significantly contribute to low-frequency self-pulsing $[\underline{4}, \underline{5}]$. However, PSINT decreases as the pump current increases, and therefore this process can induce self-pulsing only near the lasing threshold $[1,2]$. To reveal a mechanism that can drive self-pulsing with pump currents beyond the lasing threshold, we test and experimentally prove the concept of coherence and anticoherence resonance (CR and ACR) [12-14]. The details are reported in this Letter.

The experimental setup used to study self-pulsing in EDFL is shown in Fig. 1. The laser cavity consists of a high-concentration $\left(c_{\mathrm{Er}}^{-}=3.710^{25}\right.$ ions $\left./ \mathrm{m}^{3}\right)$ erbiumdoped fiber (5 m, Liekki Er40-4/125), with a Faraday mirror $(R=90 \%)$ and a fiber Bragg grating $\left(\lambda_{\text {peak }}=\right.$ $1556 \mathrm{~nm}, \Delta \lambda_{3 \mathrm{~dB}}=0.2 \mathrm{~nm}, \quad R=86 \%$ ) as reflectors. According to manufacturing data [10] and previous experimental results [11], the short-range coordination or- der of erbium ions is suppressed and no saturable absorption is present. Total intracavity losses are $5 \mathrm{~dB}$. The Faraday mirror rotates the plane of polarization of the reflected beam by $\pi / 2$ at $\lambda=1550 \mathrm{~nm}$. To pump the erbium-doped fiber, we used a $978 \mathrm{~nm}$ laser diode (LD) with a tunable current source (200-600 mA). The pump current at the lasing and self-pulsing threshold was $240 \mathrm{~mA}$.

To characterize the polarization instability as a possible source of high-frequency chaotic oscillations, we used an in-line polarimeter (Thorlabs, IPM 5300, $1 \mathrm{MHz}$ bandwidth). The states of polarization (SOP) of the laser output at pump currents 250 and $265 \mathrm{~mA}$, over a time period of $1 \mathrm{~ms}$ (1024 data points), are shown in Figs. 2(a) and 2 (b) (left) as normalized Stokes parameters $s_{1}, s_{2}$, and $s_{3}$. With noise added to the pump current (265 mA), we observed a transformation of the SOPs from a localized to almost uniform distribution [Fig. 2(c)]. The corresponding variations of the intensities of two crosspolarized modes $\left(I_{x}\right.$ and $\left.I_{y}\right)$ are shown in Fig. 2 (right). As seen in Figs. 2(a) and 2(b), the SOP fluctuations are very fast, with characteristic switching time of $1 \mu \mathrm{s}$. The fast polarization changes manifest as rapid variations of two orthogonal components of the electric field (Fig. 2, right). At the same time, low-frequency $(\sim 10 \mathrm{kHz})$

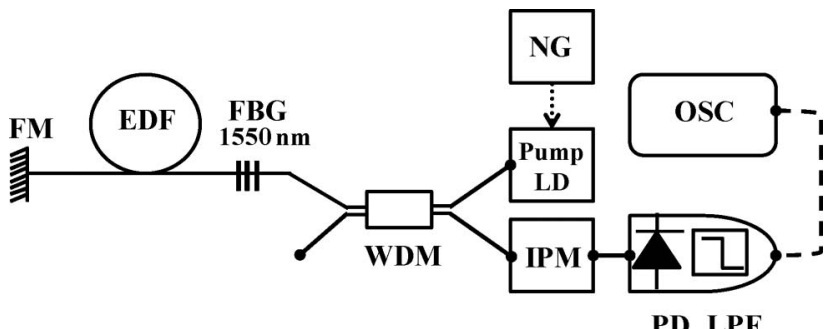

PD LPF

Fig. 1. Experimental setup: FM, Faraday mirror; EDF, highconcentration erbium-doped fiber; FBG, fiber Bragg grating; WDM, wavelength division multiplexer; Pump LD, pump laser diode; NG, noise generator; IPM, in-line polarimeter; PD LPF, photo detector with low-pass filter; OSC, oscilloscope. 

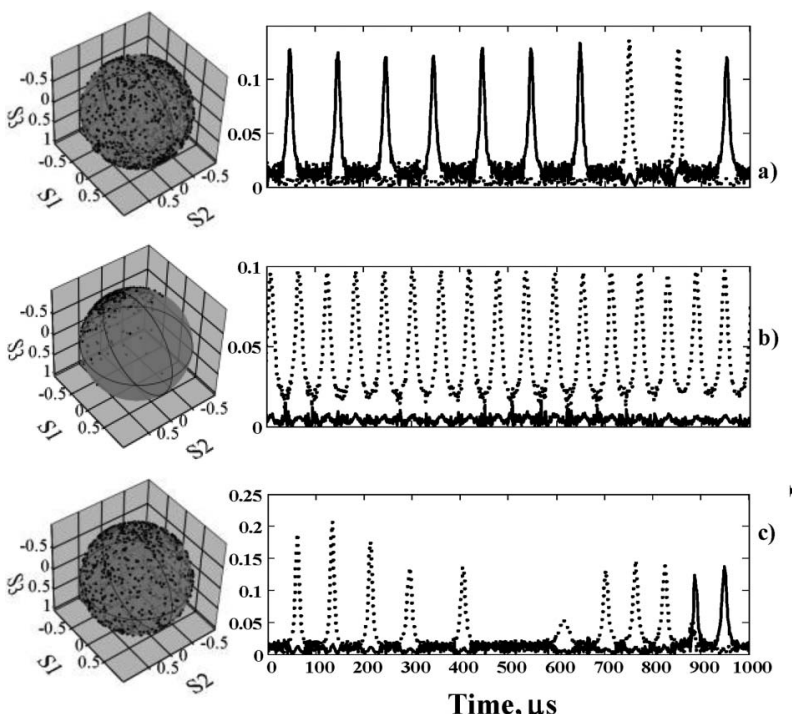

Time, $\mu$ s

Fig. 2. Fluctuating polarization of the EDFL: normalized Stokes parameters on the Poincaré sphere (left) and intensity of $x$-polarization (solid curve) and $y$-polarization (dotted curve) versus time (right). Parameters: (a) pump current $250 \mathrm{~mA}$, no additional noise; (b) pump current $265 \mathrm{~mA}$ (maximum variance of interspike intervals), no additional noise; (c) pump current $265 \mathrm{~mA}$ (suppression of intermittent self-pulsing), noise applied to pump current.

self-pulsing of the cross-polarized modes is clearly seen. With noise ( $5 \mathrm{kHz}$ bandwidth) added to the pump current, a variable modulation of the period and amplitude of pulses was observed [Fig. 2(c)]. The chaotic nature of the SOP fluctuations in EDFL has also been reported in $[2,3]$.

A related phenomenon, intermittent self-pulsing, also occurs in EDFL, but as follows from [6], this effect can be revealed on time scales of seconds. We used a photodetector, low-pass filter (New Focus, 2051-FC, $10 \mathrm{MHz}$ bandwidth, cutoff frequency $100 \mathrm{kHz}$ ), and oscilloscope (Tektronix TDS3054, $500 \mathrm{MHz}$ bandwidth) to collect data over a few seconds (Fig. 3). Intensity self-pulsing occurs at current $250 \mathrm{~mA}$ [Fig. 3(a)], with slight irregularities in amplitude and period (though the period is not resolved). For the pump current of $265 \mathrm{~mA}$, the period irregularities lead to intermittent self-pulsing [Fig. 3(b)] in the form of radiation suppressions on $1 \mathrm{~Hz}$ scale. With a noise modulation of the pump current, such self-pulsing vanishes [Fig. 3(c)]. For quantitative description of the observed low-frequency self-pulsing, we define a normalized variance of spiking intervals $R$ and intensity correlation time $T_{c}$ [12]:

$$
R=\sqrt{\left\langle t_{p}^{2}\right\rangle-\left\langle t_{p}\right\rangle^{2}} /\left\langle t_{p}\right\rangle, \quad T_{c}=\int_{0}^{\infty} C^{2}(\tau) \mathrm{d} \tau,
$$

where

$$
C(\tau)=\langle I(t) I(t+\tau)\rangle /\left\langle I^{2}(t)\right\rangle, \quad I(t)=I_{L}(t)-\left\langle I_{L}(t)\right\rangle .
$$

Here the brackets denote time average, $t_{p}$ is the fluctuating interspike interval, $I_{L}(t)$ and $I(t)$ are the lasing output intensity and associated zero-mean intensity, and $C(\tau)$ is

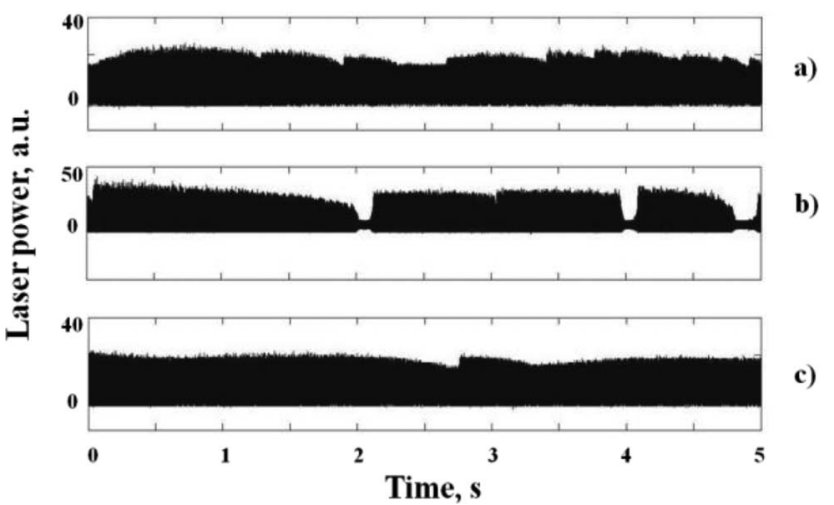

Fig. 3. (a) Self-pulsing of the EDFL intensity with $250 \mathrm{~mA}$ pump current (no noise added), (b) intermittent self-pulsing with $265 \mathrm{~mA}$ pump current (no noise added), (c) disappearance of intermittent self-pulsing with $265 \mathrm{~mA}$ pump current and added noise.

a normalized autocorrelation function. Experimental results for $R$ and $T_{c}$ as a function of the laser pump current without (solid curves) and with (dotted curves) external noise modulation are shown in Fig. 4 for the current in the range of 250-500 mA.

Several observations can be made from Fig. 4 . The variance $R$ increases as the current varies from $\overline{2} 50 \mathrm{~mA}$ to $265 \mathrm{~mA}$. The latter value corresponds to the large variations of the interspike intervals [Fig. 3(b)], i.e., the case of intermittent self-pulsing. Similar behavior was observed for ring cavity EDFLs in [6]. At this pump current, the correlation time $T_{c}$ takes its minimum. Further increase of the pump current leads to the minimum of $R$ and the maximum of $T_{c}$ at $280 \mathrm{~mA}$. According to [4,5], noise added in the pump current increases random laser output variations through PSINT. Hence, the distribution of interspike intervals approaches homogeneous distribution with variance of one-third. Also, the increased random amplitude modulation results in suppressed correlation time $T_{c}$. The pump power fluctuation is shown in the inset in Fig. 4.

To treat the results in terms of coherence resonance, one needs to identify the noise source that drives the amplitude and spiking intervals of intensity self-pulsing,

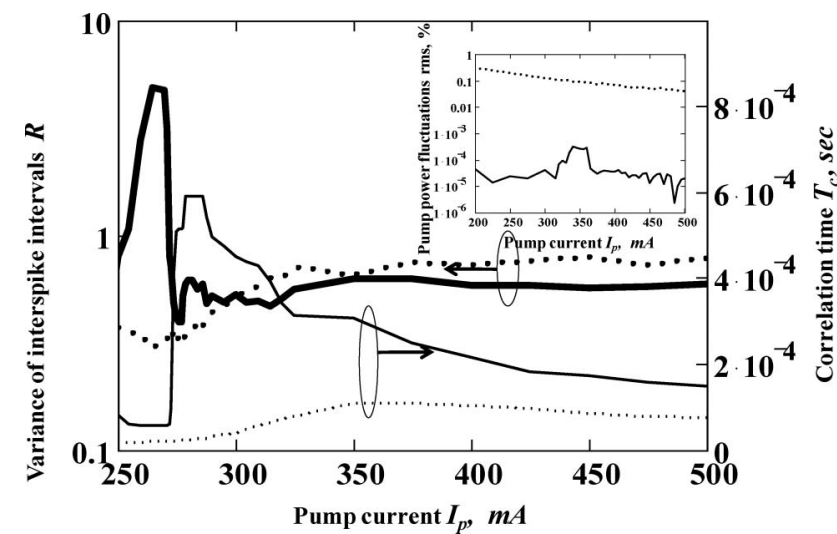

Fig. 4. Normalized variance of interspike intervals $R$ (thick solid and dotted curves) and correlation time $T_{c}$ (thin solid and dotted curves) as a function of the pump current. Inset, pump power fluctuations rms versus pump current. No noise (solid curve) and additional noise (dotted curve) applied to the pump current. 
which depends on the pump current. It is known that for currents above the lasing threshold, the population of the first excited level of erbium is clamped, and so the average amplitude of spontaneous emission noise also is clamped [13]. However, if the laser dynamics shows the presence of a spiral Shilnikov attractor, the clamped spontaneous emission noise transforms it to fast chaotic oscillations [15]. It is likely that such an attractor is typical for EDFL's polarization dynamics, as in dye lasers [15]. In analogy to results in [15], the threshold value for this type of chaos can be found as $\mu_{1}=\gamma /(2 k)$, where $k$ and $\gamma$ are the relaxation rates for cavity photons and population inversion, respectively, $\mu_{1}=I_{p} / I_{p \text {,th }}-1$, and $I_{p}$ and $I_{p, \text { th }}$ are the pump current and its threshold value. For EDFL, $\gamma=10^{2} \mathrm{~s}^{-1}$, $k=10^{7} \mathrm{~s}^{-1}$, and so $\mu_{1} \approx 0$; i.e., the first threshold coincides with the threshold of polarization chaos. Because of limited sampling rate of polarimeter $(1 \mathrm{MHz})$, we cannot observe polarization dynamics, including polarization chaos, on ns scale as was done in [1-3]. We simply show that fast chaotic switching between cross-polarized components occurs in the range of few microseconds. In addition to polarization instabilities, multimode instability resulting in spontaneous mode locking can also lead to high-frequency chaotic oscillations $[6,16]$. The threshold value of such instability is $\mu_{1} \approx 0.05$, which is quite close to the threshold of polarization instability [6]. It is likely that nanosecond-scale chaotic dynamics can be caused by combination of multimode and polarization instabilities. The amplitude of such fluctuations is proportional to the pump current [3]. So extrema in coherence resonance signatures, viz., the intensity correlation time and the variance of interspike intervals, as functions of the pump current, indicate the presence of deterministic coherence resonance due to deterministic origin of highfrequency chaotic oscillations [13].

Because of proximity to the threshold, intermittent self-pulsing [Fig. 3(b)] and growing variance $R$ with increased pump current (Fig. 4) can be driven by spontaneous noise rather than by deterministic chaos due to multimode instabilities $[6,16]$. The spectral gain of EDFL is flat, and the operating mode at the threshold is not defined. Coinciding thresholds for single- and multimode lasing can result in hysteresis and hopping between these regimes driven by spontaneous noise (amplified spontaneous emission and noise caused by PSINT) [6]. The total intensity of multimode operation depends on the phase difference between modes, and can be very low []]. Be- cause the maximum of the variance $R$ coincides with the minimum of the correlation time $T_{c}$ for intermittent self-pulsing, the effect can be called anticoherence resonance [14].

In conclusion, we have shown that fast intensity fluctuations caused by multimode and polarization instabilities play the role of an external noise that leads to low-frequency ( $10 \mathrm{kHz})$ self-pulsing of the output intensity in a high-concentration erbium-doped fiber laser through a deterministic coherence resonance scenario.

S. Sergeyev acknowledges financial support from the European Union (EU) program FP7-PEOPLE-2009-IEF (grant 253297); A. T. Friberg thanks the Academy of Finland (grant 128331).

\section{References}

1. G. D. Van Wiggeren and R. Roy, Phys. Rev. Lett. 88, 097903 (2002).

2. G. D. Van Wiggeren and R. Roy, Opt. Commun. 164, 107 (1999).

3. Q. L. Williams, J. Garcia-Ojalvo, and R. Roy, Phys. Rev. A 55, 2376 (1997).

4. Yu. O. Barmenkov and A. V. Kir'yanov, Opt. Express 12, 3171 (2004).

5. S. Sergeyev, K. O’Mahoney, S. Popov, and A. T. Friberg, Central Eur. J. Phys. 8, 159 (2010).

6. T. Voigt, M. O. Lenz, F. Mitschke, E. Roldan, and G. J. de Valcarcel, Appl. Phys. B 79, 175 (2004).

7. P. L. Boudec, P. L. Francois, E. Delevaque, J. F. Bayon, F. Sanchez, and G. Stephan, Opt. Quantum Electron. 25, 501 (1993).

8. P. M. Peters and S. N. Houde-Walter, J. Non-Cryst. Solids 239, 162 (1998).

9. J. Lægsgaard, Phys. Rev. B 65, 174114 (2002).

10. S. Tammela, M. Hotoleanu, P. Kiiveri, H. Valkonen, S. Sarkilahti, and K. Janka, in Conference on Optical Fiber Communications, Vol. 1, 2003 OSA Technical Digest Series (Optical Society of America, 2003), pp. 376-377.

11. S. Sergeyev and D. Khoptyar, Proc. SPIE 6610, 66100L (2007).

12. S. Pikovsky and J. Kurths, Phys. Rev. Lett. 78, 775 (1997).

13. J. F. Martinez Avila, H. L. D. de S. Cavalcante, and J. R. Rios Leite, Phys. Rev. Lett. 93, 144101 (2004).

14. M. Lacasta, F. Sagues, and J. M. Sancho, Phys. Rev. E 66, 045105 (2002).

15. S. Sergeyev, Phys. Rev. A 59, 3909 (1999).

16. P. Chenkosol and L. W. Casperson, J. Opt. Soc. Am. B 24, 1199 (2007). 